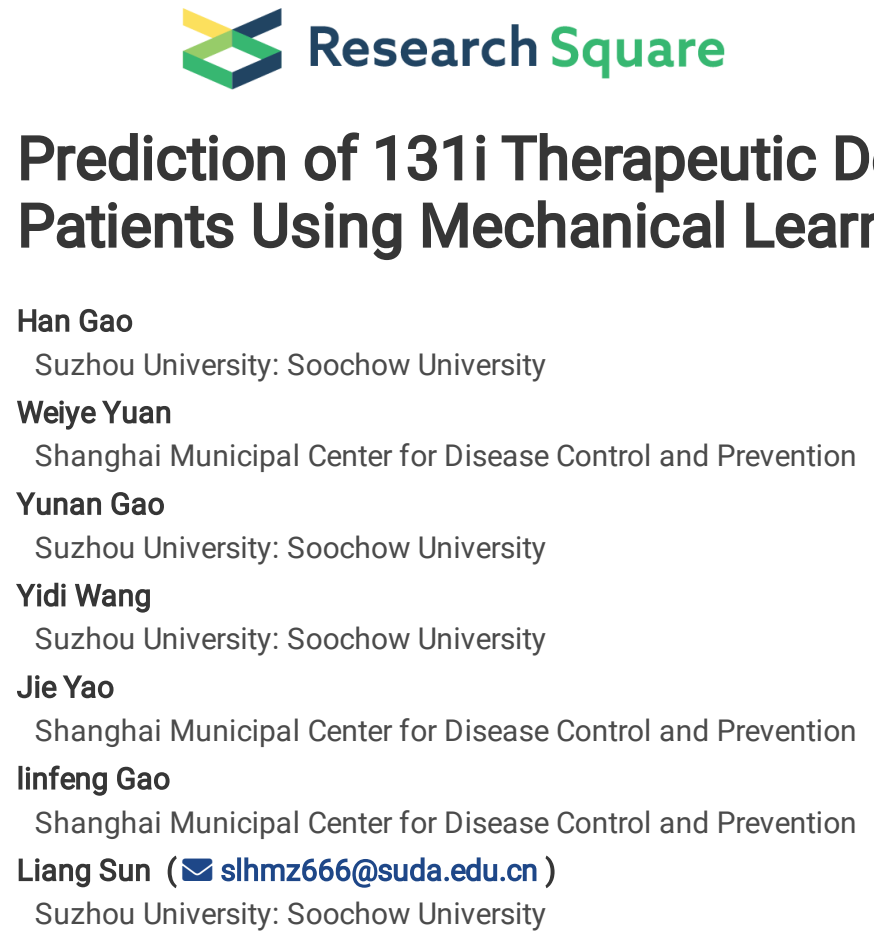

\title{
Prediction of 131i Therapeutic Dose and Prognosis in Hyperthyroidism Patients Using Mechanical Learning Model
}




\section{Abstract}

Objective

Multiple mechanical learning models were used to predict the therapeutic dose of ${ }^{131}$ I radionuclide in patients with hyperthyroidism, and to compare the calculation results of each prediction model to obtain the optimal model for dose prediction. Meanwhile, the classification model was used to classify the prognosis of the existing clinical hyperthyroidism case data in order to evaluate the administration results and provide reference for the dose given by clinicians.

Methods

According to the data of hyperthyroidism patients treated with ${ }^{131} \mathrm{I}$ in nuclear medicine department of many hospitals, a prediction model was established based on MATLAB. Firstly, the prediction results of BP neural network, radial basis function (RBF) neural network and support vector machine (SVM) were compared with small sample data, and then the optimal model was selected to predict the drug dose. BP-AdaBoost, SVM and random forest were used to classify the patients after recovery and evaluate whether the dose was accurate.

Results

The average errors of BP neural network, RBF neural network and SVM models trained with small samples were $6.58 \%, 17.25 \%$ and $14.09 \%$ respectively. After comparison, BP neural network was selected to establish the prediction model. The data of 30 cases were randomly selected to verify BP neural network, and average error of the prediction results was $11.99 \%$. Using SVM, BP-AdaBoost and random forest models, 100 groups of case data were selected as the training set and 10 groups as the test set. The classification accuracy were $80 \%, 90 \%$ and $100 \%$ respectively. The random forest model with the highest accuracy was selected as the large sample prediction. When 318 groups of cases were trained and 35 groups of cases were used for the test, the classification accuracy was $97.14 \%$.

\section{Conclusion}

This study compared the prediction effects of various prediction models on ${ }^{131}$ I therapeutic dose in patients with hyperthyroidism and the accuracy of prognosis classification. BP neural network and random forest achieved the best results respectively. The two models provide reference for clinicians when giving the dose, which has clinical practical significance.

\section{Introduction}

Hyperthyroidism is a common thyroid disease. At present, the commonly used clinical treatment methods include radioactive iodine ( $\left.{ }^{131} \mathrm{I}\right)$ treatment, antithyroid drug (ATD), and thyroidectomy ${ }^{[1]}$. Radioactive iodine ${ }^{131}$ I therapy was introduced in the 1940 s $^{[2,3]}$. After ${ }^{131}$ I enters the human body, it targets the thyroid gland, and has less distribution in other tissues and organs with short residence time, therefore, the radiation dose of conventional dose ${ }^{131}$ I to gonad, bone marrow and liver is low, and generally will not cause serious radiation damage. ${ }^{131}$ I decay release $\beta$ radiation efficiently destroys thyroid follicular cells, so it is especially suitable for patients with poor effect of ATD treatment, repeated recurrence, adverse reactions of ATD treatment, combined with liver function injury and unable to tolerate surgery. Compared with thyroidectomy and ATD, radionuclide therapy for hyperthyroidism has the advantages of convenient operation, shorter time needed to control the symptoms of hyperthyroidism, avoiding the risk caused by surgery, adverse reactions of thyroid drugs and low recurrence rate ${ }^{[4]}$. Clinically, ${ }^{131}$ I dose of hyperthyroidism patients is often determined by fixed formula calculation combined with doctors' empirical medication ${ }^{[5]}$, and there may be great differences in the dose of the same patient by different doctors. In addition, there are some problems in ${ }^{131}$ I treatment, such as inaccurate measurement of biodynamics, unclear and unquantifiable individual radiosensitivity ${ }^{[6]}$, potential confounding factors ${ }^{[7]}$ interference, which are bound to bring difficulties to the personalized calculation of dose for patients with hyperthyroidism.

Based on the doctor's clinical experience, thyroid size, texture, heart rate and other factors will affect the ${ }^{131}$ I therapeutic dose. However, there is few study found to point out the specific coefficients corresponding to the above factors in dose calculation. For a new case, it is impossible to directly calculate the actual ${ }^{131}$ I therapeutic dose required by the case with the above parameters, so it is difficult to predict the treatment effect of the patient. The machine learning model represented by artificial neural network model can convert information to higher dimensional representation, and it could realize the classification or prediction of complex variable relations, which is impossible for traditional statistical models (such as logistic regression model) ${ }^{[8]} \rrbracket$ therefore, machine learning model is becoming more and more important in the field of prediction modeling ${ }^{\left[{ }^{8-10]}\right.}$. Commonly used machine learning models include SVM, random forests ${ }^{[11]}$ and etc. besides neural network, which are widely applied in clinical medicine, such as the diagnosis of skin cancer ${ }^{[12]}$, chronic kidney disease ${ }^{[13]}$, breast cancer ${ }^{[14]}$, and other common diseases ${ }^{[15-}$ 17]. There are also some researches for thyroid diseases ${ }^{[18-20]}$, however, the recognition of thyroid images is mainly based on convolutional neural network with few discussion on ${ }^{131}$ I therapeutic dose and prognosis evaluation. 
Therefore, models for predicting the dose of ${ }^{131}$ I hyperthyroidism radionuclide therapy and classifying the prognosis are proposed in this paper. Based on clinical medical research and doctor's suggestions on hyperthyroidism, indexes of thyroid weight (TW), free triiodothyronine (FT3), free thyroxine (FT4), thyroid stimulating hormone (TSH) and heart rate (HR) and so on were selected, 8 indexes were used for dose prediction, 11 indexes were used for classification, and the prediction model was established by Matlab platform. The dose of ${ }^{131}$ I nuclide in the treatment of hyperthyroidism was predicted, and the prognosis was evaluated by classification model.

\section{Materials And Methods}

\section{Clinical data}

353 cases of thyroid treated with ${ }^{131}$ I and blood examination reports, including basic information, disease characteristics, pathological characteristics, treatment and prognosis, were collected from the medical record management system of the information center of various hospitals in Shanghai. The case data directly collected included 17 indicators, such as age, course of disease, degree of goiter, iodine uptake rate and thyroid quality.

\section{Data processing}

Firstly, the collected cases were screened. According to the 2016 edition of diagnosis and treatment guidelines of the American Thyroid Association (ATA) ${ }^{[21]}$, the patients' thyroid function returned to normal and hypothyroidism were considered successful after ${ }^{131}$ I treatment. Therefore, the effective dose of this study was "cure", "improvement" and "hypothyroidism". According to the clinical experience, the case indexes were preliminarily screened, and 8 indexes including age, course of disease, degree of goiter, HR, TW, FT3, FT4 and 24-hour iodine uptake rate were selected as effective indexes. In order to avoid the neural network taking too long in the training process and reduce the prediction error, the data and the input data were normalized in the $[0,1]$ interval by using the "mapminmax" code of statistical MATLAB.

\section{Model selection}

1) BP neural network

BP neural network is a multilayer feedforward neural network based on the principle of error back propagation. It is composed of input layer, hidden layer and output layer. The weight and threshold ${ }^{[22,23]}$ is adjusted layer by layer according to the error. It can learn and store a large number of input-output mode mapping relationships without revealing the mathematical equations describing this mapping relationship in advance, which is a very effective tool for nonlinear regression prediction ${ }^{[24]}$. Although the single hidden layer network trained for many times in theory can approximate any rational function with a sufficient number of samples, for complex samples with multiple inputs, multiple hidden layers can be used to reduce the prediction error $[22,25,26]$. The topology of the neural network model with structure 8-9-9-1 used in this paper is shown in Figure 1 (a), where $\omega_{\mathrm{ij}}$ represents the weight of neural network connection. The neural network is established by using MATLAB "newff" code, the training times is set to 100 , the learning rate is set to 0.005 , and the "trainlm" code is used for training.

2) RBF neural network

RBF network is a three-layer forward neural network proposed by Broomhead et al. [27] according to the idea of multi-dimensional space interpolation. The first layer is the input layer, the second layer is the hidden layer, and its activation function is a non-negative nonlinear function that is radial symmetric of center point, and it is decreasing. The third layer is the output layer. The main principle is to transform the input vector through the radial basis function to make it linearly separable in the high-dimensional space. The network structure selected in this paper is shown in Figure 1 (b) ${ }^{[27]}$. RBF network has simple structure, fast convergence and excellent generalization ability, and is widely used in the field of prediction and analysis ${ }^{[28,29]}$. In this paper, "newrb" code in MATLAB is used to establish neural network, and "SPREAD" parameter set to 0.12.

\section{$3 \llbracket S V M$}

SVM is a machine learning model based on learning statistical theory, which takes into account training error and generalization ability, and shows

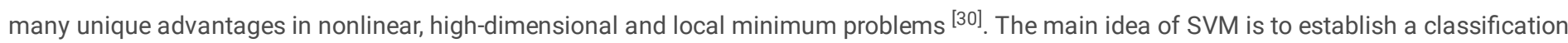
hyperplane as the decision surface to maximize the isolation edge between positive and negative examples. The structures of regression and prediction are shown in Fig. 1 (c) and 2 (a) ${ }^{[31]}$, where $\mathrm{K}\left(\mathrm{x}_{\mathrm{i}}, \mathrm{x}\right)$ represents its kernel function. In recent years, SVM based regression prediction has been used in the medical field ${ }^{[30,32]}$, and its application in medical diagnosis is gradually increasing ${ }^{[33]}$. Based on libsvm toolbox ${ }^{[34]}$, the ${ }^{131}$ I therapeutic dose model was established, and the input parameters of SVM were determined by cross validation method. The parameters used for prediction and classification were $c=7.0267, g=35.6503, p=0.16218, s=3 ; c=67.43, g=4.31, p=0.149, s=0$ [

4『BP-AdaBoost model 
The basic idea of BP-AdaBoost model is to combine the outputs of multiple weak classifiers to produce effective classification. The neural network classification model is used as the weak classifier of AdaBoost algorithm, the BP neural network is repeatedly trained to predict the sample output,

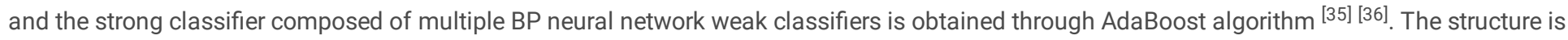
shown in Fig. 2 (a).

\section{5囚Random forest}

Random forest model is a decision treesbased algorithm ${ }^{[11]}$ that combines the idea of "bootstrap aggregating" [37] and the "random subspace" method ${ }^{[38]}$. It improves the prediction accuracy of the model by summarizing a large number of classification trees. The essence of the algorithm is a classifier containing multiple decision trees. The formation of these decision trees adopts the random method, which greatly increases the prediction accuracy without increasing the amount of computation. The structure is shown in Fig. 2 (b). It has been used in disease classification, diagnosis and classification ${ }^{[39]}$, ecological science ${ }^{[40]}$ and other fields. This study uses the randomforest-matlab ${ }^{[41]}$ open source toolbox developed by the University of Colorado Boulder to classify the prognosis.

\section{Results}

\section{Dose prediction model}

In order to analyze the prediction effects of different algorithms, 100 groups of samples were first randomly selected as the training set, and 10 groups were randomly selected from the remaining samples as the test set to compare their prediction results. In order to ensure the randomness of sample extraction, 308 groups of effective samples were randomly arranged by using the "randperm" code of MATLAB. The first 100 groups were selected as the training set and the 101 to 110 groups were selected as the test set. After calculation, the average errors of BP neural network, RBF neural network and SVM model for the prediction of the same test set were $6.58 \%, 17.25 \%$ and $14.09 \%$ respectively. The specific results are shown in Table 1.

Figure 3 shows the validation relative errors of the three models. The predicted values of the three models are very close to the clinical data values, and the prediction ability is very good. From the 10 groups of validation data, it can be seen that BP network has greater advantages in prediction accuracy than RBF neural network and SVM, most of the deviations are within $10 \%$, and the prediction accuracy is good. On the whole, it meets the needs of model application. To sum up, BP network was finally chosen for prediction.

The overall training process of BP neural network is shown in Fig. 4. The data is divided into three parts by Matlab: "training", "validation" and "test". The "training" sample is used to train the network, "validation" sample is used to debug network parameters, and the "test" sample is used as evaluation to test the fitting effect. "Goal " is set by the "net trainparam Goal" parameter, which represents the expected minimum error". "Best" is the error accuracy actually obtained after training ${ }^{[24]}$. As can be seen from Figure 4 (a), the error of the test network decreases rapidly when the number of iterations is 1-5, but with the progress of training and the increase of the number of iterations, the network error trend gradually flattens. When the number of iterations is 20 times, the optimal solution appears. Figure 4 (b) reflects the fitting effect in the training set. The closer the $\mathrm{R}$ value is to 1 , the better the fitting effect of the model. It can be seen that the model has better prediction and regression ability.

The output results of the BP neural network model were verified by clinical data. The training set and test set of this study were divided by $9: 1$, that is, one tenth of the total sample size (31 groups of data) was selected for verification. The comparison results between the output values and clinical data are shown in table 2 and figure 5 (Table 2 is limited to 10 groups of data due to space limitation), and the average error of the prediction results was $11.99 \%$. It can be seen from Figure 5 that the predicted data is very close to the verified data. It can be considered that the model has the ability to describe the mapping relationship between blood test indexes, throid weight, HR and dose, and can predict ${ }^{131}$ I therapeutic dose value to a certain extent.

\section{Prognostic classification model}

Compared with the prediction model, the classification model takes the dose as the input parameter to evaluate its effectiveness. In this study, "cure", "improvement" and "hypothyroidism" were taken as effective dose, represented by "1", and "hyperthyroidism" and "recurrence" were taken as ineffective dose, represented by "0". In order to compare the classification effects of SVM, BP-AdaBoost and random forest models, 100 groups of samples were first randomly selected as the training set and 10 groups from the remaining samples as the test set to compare the results.

For SVM model, the S parameter was set to 0 , indicating that the classification model was adopted; $T$ parameter was set to 1 , indicating the use of linear kernel function; The $c$ and g parameter was optimized by using the "gasvmcgpforregress" code built in the toolbox, and the value was as described above. The weak classifier of BP-AdaBoost model adopted a single hidden layer BP network with hidden neurons of 6 . A total of 11 weak classifiers were trained and generated. Finally, the strong classifier composed of 11 weak classifiers was used for prediction. For the random forest model, the number of decision trees had a great impact on the accuracy of classification results. In this study, the optimal value was obtained by cyclic comparison between 1-100. 
For the 10 validation sets, the classification accuracy of SVM, BP-AdaBoost and random forest models were $80 \%, 90 \%$ and $100 \%$ respectively. The accuracy of random forest classification was the highest, and all 10 groups of test set samples could be classified correctly. After that, 353 groups of all samples were divided into training set and test set according to the ratio of 9:1 for training. In order to obtain the optimal number of decision trees, this study compared the number of 5-100 decision trees. The results are shown in Figure 6 . With the increase of decision trees, the accuracy is finally stable at $\mathbf{9 7 . 1 4 \%}$, with better accuracy. The specific data of the test set are shown in Table 3 , and only 10 cases are shown for length reasons.

A total of 353 cases were collected in this study, but only 40 cases of hyperthyroidism (poor prognosis, recurrence) and the remaining 313 cases were cured (hypothyroidism, improvement). Although the classification model established according to this sample performed well, it was relatively insensitive to hyperthyroidism because there were less hyperthyroidism results, resulting in high accuracy. There were two reasons for the excessive hypothyroidism data in the sample: one was that the ${ }^{131}$ I dose treatment received by most patients was effective; the other was that the patient follow-up data was not complete and the follow-up time was too short. The above factors would lead to unbalanced data. The follow-up mechanism should be further improved and more sample data should be included for training, and then the model can be more in line with the actual situation, which will be the focus of the next step of this study.

\section{Conclusion}

How to accurately calculate the optimal dose of ${ }^{131}$ I has always been a difficult problem in the treatment of hyperthyroidism. At present, the commonly used fixed dose method ${ }^{[42]}$ and adjusted dose method ${ }^{[43]}$ are still controversial ${ }^{[44]}$. This study proposes a prediction model based on artificial intelligence to provide reference for clinicians. According to the patient's physical condition, blood routine test, medical history and course of disease, the optimal ${ }^{131}$ I dose can be predicted. The data of this study samples are collected from many hospitals, with a wide range of sources and a wide range of age and course of disease, covering all stages of the course of hyperthyroidism. By comparing the prediction effect of BP neural network, RBF neural network and SVM, and the classification effect of SVM, BP-AdaBoost and random forest, it is found that the prediction error of BP neural network is the smallest and the classification accuracy of random forest is the highest. Therefore, BP neural network is finally selected as the prediction model and random forest as the classification model.

The purpose of this study is to accurately calculate the therapeutic dose of radioactive iodine in patients with hyperthyroidism and predict the prognosis of hyperthyroidism. Through the simulation of prediction model and classification model, it can provide reference for clinicians to give drugs, and try to avoid hypothyroidism caused by insufficient or excessive administration. At the same time, the machine learning model has the characteristics of more training samples and higher prediction accuracy, which makes machine learning more potential in the era of big data [45]. After training, the model has good accuracy and can basically predict the dose. At the same time, compared with the traditional formula method, it also has the advantages of simple operation and strong repeatability. It can meet the needs of patients, staff and the public for radiation protection and reduce the dose difference caused by doctors' personal reasons. The future work of this study is to further expand the data samples, improve the prediction accuracy, and apply the model to clinical practice to test the prediction results.

\section{Declarations}

\section{Funding}

This work was funding by Collaborative Innovation Center of Radiological Medicine of Jiangsu Higher Education Institutions; China funding of the National Natural Science Foundation of China, China(Grant No. 11575124); the Priority Academic Program Development of Jiangsu Higher Education Institutions (PAPD) China; and the Nuclear Energy Development Project, China (No.2016-1295).Shanghai Public Health Key Discipline Construction Plan Project (GWV-10.1-XK10). Excellent Academic Leader Program of Shanghai Three-year Action Plan for Public Health System Construction (GWV-10.2-XD16)

\section{References}

1. Fadeyev VV, Karseladse EA. Hyperthyroidism and Other Causes of Thyrotoxicosis: Management Guidelines of the American Thyroid Association and American Association of Clinical Endocrinologists. Clinical and experimental thyroidology. 2011;7(4):8.

2. Joop BS, Laszlo H. Radioiodine Therapy in Benign Thyroid Diseases: Effects, Side Effects, and Factors Affecting Therapeutic Outcome. Endocrine Reviews. 2012;33(6):920-80.

3. Aktolun C, Urhan M. Radioiodine Therapy of Benign Thyroid Diseases: Graves' Disease, Plummer's Disease, Non-toxic Goiter and Nodules. Springer New York. 2013.

4. Clinical nursing of (131)I therapyin patients with hyperthyroidism. Journal of Medical Imaging. 2005. 
5. Willegaignon J, Sapienza MT, Buchpiguel CA. Radioiodine therapy for Graves disease: thyroid absorbed dose of 300 Gy-tuning the target for therapy planning. Clinical Nuclear Medicine. 2013;38(4):231-6.

6. Bonnema SJ. On the factors affecting therapeutic outcome from radioiodine therapy in benign thyroid diseases. 2012.

7. NYGAARD B, FABER J, VEJE A, HEGEDÜS L, HANSEN JM. Transition of nodular toxic goiter to autoimmune hyperthyroidism triggered by 131 therapy. Thyroid. 1999;9(5):477-81.

8. Renganathan V. Overview of artificial neural network models in the biomedical domain. Bratislavske lekarske listy. 2019;120(7):536-40.

9. Ajjolli Nagaraja A, Fontaine N, Delsaut M, Charton P, Damour C, Offmann B, et al. Flux prediction using artificial neural network (ANN) for the upper part of glycolysis. PloS one. 2019;14(5):e0216178.

10. Zhang Z. A gentle introduction to artificial neural networks. Annals of translational medicine. 2016;4(19).

11. Breiman. Random forests. MACH LEARN. 2001;2001,45(1)(-):5-32.

12. Zhang N, Cai Y-X, Wang Y-Y, Tian Y-T, Wang X-L, Badami B. Skin cancer diagnosis based on optimized convolutional neural network. Artificial intelligence in medicine. 2020;102:101756.

13. Ma F, Sun T, Liu L, Jing H. Detection and diagnosis of chronic kidney disease using deep learning-based heterogeneous modified artificial neural network. Future Generation Computer Systems. 2020;111:17-26.

14. Lim TS, Tay KG, Huong A, Lim XY. Breast cancer diagnosis system using hybrid support vector machine-artificial neural network. International Journal of Electrical \& Computer Engineering (2088-8708). 2021;11(4).

15. Yong, Qi, Haozhe, Liu, Wentian, Zhang, et al., editors. A Classification Diagnosis of Liver Medical Data Based on Various Artificial Neural Networks2018.

16. Shamsheyeva A, Sowmya A, editors. The anisotropic Gaussian kernel for SVM classification of HRCT images of the lung. Intelligent Sensors, Sensor Networks \& Information Processing Conference; 2005.

17. Vogado, Luis, H., S., Veras, Rodrigo, et al. Leukemia diagnosis in blood slides using transfer learning in CNNs and SVM for classification. Engineering Applications of Artificial Intelligence the International Journal of Intelligent Real Time Automation. 2018.

18. Ozyilmaz L, Yildirim T, editors. Diagnosis of thyroid disease using artificial neural network methods. International Conference on Neural Information Processing; 2002.

19. Lahner E, Intraligi M, Buscema M, Centanni M, Vannella L, Grossi E, et al. Artificial neural networks in the recognition of the presence of thyroid disease in patients with atrophic body gastritis. World J Gastroenterol. 2008;14(4):563-8.

20. Shukla A, Tiwari R, Kaur P, Janghel RR, editors. Diagnosis of Thyroid Disorders using Artificial Neural Networks. IEEE International Advance Computing Conference; 2009.

21. Ross DS, Burch HB, Cooper DS, Greenlee MC, Laurberg P, Maia AL, et al. 2016 American Thyroid Association guidelines for diagnosis and management of hyperthyroidism and other causes of thyrotoxicosis. Thyroid. 2016;26(10):1343-421.

22. Hecht-nielsen R. Kolmogorov"s Mapping Neural Network Existence Theorem. Proc First IEEE Intern Conf on Nenral Nets. 1987/01/01;1.

23. Sadeghi B. A BP-neural network predictor model for plastic injection molding process. Journal of Materials Processing Technology. 2000;103(3):411-6.

24. Gao H, Li X, Li Z, Wang Y, Gao Y, Tang W, et al. The buildup factor calculations of concrete with different proportions of CRT based on a BP neural network by MCNP. Journal of Nuclear Science and Technology. 2021;58(6):667-75.

25. Hornik K. Approximation capabilities of multilayer feedforward networks. Neural Networks. 1991;4(2):251-7.

26. Zeping W. Prediction and comparative analysis of water quality based on GA-BP and multi-hidden-layer BP network model [J]. Journal of Water Resources and Water Engineering. 2013;3.

27. Tudu B, Jana A, Metla A, Ghosh D, Bhattacharyya N, Bandyopadhyay R. Electronic nose for black tea quality evaluation by an incremental RBF network. Sensors \& Actuators B Chemical. 2009;138(1):90-5.

28. Park J, Sandberg I. Universal Approximation Using Radial-Basis-Function Networks. Neural Computation. 2014;3(2):246-57. 
30. Zhang S, Zhou LI, Xin X. Support vector machine-assisted diagnosis of human malignant gastric tissues based on dielectric properties. Journal of Southern Medical University. 2017.

31. Hamiane M, Saeed F. SVM Classification of MRI Brain Images for Computer-Assisted Diagnosis. International Journal of Electrical and Computer Engineering. 2017;7(5):375-81.

32. Zhang J-f, Jiang L-d, Zhang Y-I. [Application of support vector machine approach in studying nephron toxicity of Chinese medicinal materials]. Zhongguo Zhong Yao Za Zhi. 2015;40(6):1134-8.

33. Furey, T., S., Cristianini, N., Duffy, et al. Support vector machine classification and validation of cancer tissue samples using microarray expression data. BIOINFORMATICS -OXFORD-. 2000.

34. Chang CC, Lin CJ. LIBSVM. http://wwwcsientuedutw/[Cjlin/libsvm. 2011.

35. Zhang T, Hao X, Zhang Y, Zhang M. A medical diagnosis model based on BP-AsymBoost algorithm. Xitong Gongcheng Lilun yu Shijian/System Engineering Theory and Practice. 2017;37(6):1654-64.

36. Santos S, Barros R. Online AdaBoost-based methods for multiclass problems. Artificial Intelligence Review. 2020;53(2):1293-322.

37. Santana Jr O. Bagging Predictors.

38. Tin, Kam. The random subspace method for constructing decision forests. IEEE Transactions on Pattern Analysis \& Machine Intelligence. 1998;20(8):832-.

39. Hsieh CH, Lu RH, Lee NH, Chiu WT, Li Y. Novel solutions for an old disease: Diagnosis of acute appendicitis with random forest, support vector machines, and artificial neural networks. Surgery. 2010;149(1):87-93.

40. Froeschke, JT, BF. Spatio-temporal predictive model based on environmental factors for juvenile spotted seatrout in Texas estuaries using boosted regression trees. FISH RES. 2011;2011,111(3)(-):131-8.

41. Jaiantilal A. Randomforest-matlab. Dostopno na: https://code google com/p/randomforest-matlab. $2010 ; 12$.

42. Franklyn JA, Daykin J, Holder R, Sheppard MC. Radioiodine therapy compared in patients with toxic nodular or Graves' hyperthyroidism. Qjm. 1995(3):175-80.

43. Kung A, Choi P, Lam K, Pun K, Wang C, Yeung R. Discriminant factors affecting early outcome of radioiodine treatment for Graves' disease. Clinical Radiology. 1990;42(1):52-4.

44. Hou ZF, Yao P, Tan QL. A Comparison of lodine Doses in Graves' Hyperthyroidism. Journal of Hubei Institute For Nationalities Medical Edition. 2003.

45. Zhang Y, Guo Q, Wang J. Big Data Analysis Using Neural Networks. Advanced Engineering Sciences. 2017.

\section{Tables}

Tab.1 The prediction results of three different algorithms under the same parameter 


\begin{tabular}{|c|c|c|c|c|}
\hline$T W \llbracket g \rrbracket$ & Actual value $₫ 10^{8} \mathrm{~Bq} \rrbracket$ & Predictive value-BP $₫ 10^{8} \mathrm{~Bq} \rrbracket$ & Predictive value-SVM $₫ 10^{8} \mathrm{~Bq} \rrbracket$ & Predictive value-RBF $\llbracket 10^{8} \mathrm{~Bq} \rrbracket$ \\
\hline 30.37 & 1.40 & 1.54 & 1.20 & 1.77 \\
\hline 64.6 & 3.14 & 3.09 & 3.92 & 1.98 \\
\hline 39.73 & 3.70 & 3.36 & 3.52 & 3.05 \\
\hline 24.96 & 1.66 & 1.65 & 1.48 & 1.69 \\
\hline 49.03 & 1.48 & 1.50 & 1.64 & 1.55 \\
\hline 45 & 3.33 & 3.30 & 3.29 & 2.08 \\
\hline 27.16 & 3.70 & 3.36 & 4.08 & 2.76 \\
\hline 18.77 & 1.85 & 1.57 & 1.52 & 1.86 \\
\hline 35 & 2.04 & 2.17 & 3.15 & 2.09 \\
\hline 40 & 2.22 & 2.48 & 2.01 & 1.79 \\
\hline
\end{tabular}

Tab.2 The BP network compares predicted values to actual values

\begin{tabular}{|c|c|c|c|c|c|c|c|c|c|c|}
\hline TW(g) & $\begin{array}{l}\text { HR(Times } \\
\text { / minute) }\end{array}$ & FT3(pmol/L) & FT4(pmol/L) & $\begin{array}{l}\text { Degree } \\
\text { of } \\
\text { goiter(\%) }\end{array}$ & $\begin{array}{l}\text { Course of } \\
\text { disease(month) }\end{array}$ & Age & $\begin{array}{l}\text { 24-hour } \\
\text { iodine } \\
\text { uptake } \\
\text { rate(\%) }\end{array}$ & $\begin{array}{l}\text { Actual } \\
\text { value } \\
₫ 10^{8} \mathrm{~Bq} \rrbracket\end{array}$ & $\begin{array}{l}\text { Predictive } \\
\text { value } \\
\text { } 10^{8} \mathrm{~Bq} \rrbracket\end{array}$ & Error \\
\hline 45 & 78 & 8.0 & 28.8 & 2 & 36 & 42 & 71.3 & 8.4 & 10.0 & $15.56 \%$ \\
\hline 35 & 84 & 15.6 & 53.7 & 1 & 60 & 73 & 85.2 & 6.5 & 9.0 & $27.81 \%$ \\
\hline 45 & 80 & 30.8 & 65.0 & 1 & 6 & 62 & 67.3 & 7.4 & 7.0 & $5.77 \%$ \\
\hline 50 & 84 & 15.5 & 58.6 & 1 & 6 & 42 & 57.58 & 9.9 & 10.0 & $1.03 \%$ \\
\hline 50 & 88 & 8.2 & 24.1 & 2 & 30 & 56 & 62.2 & 9.8 & 10.0 & $2.07 \%$ \\
\hline 49 & 88 & 18.0 & 5.2 & 2 & 4 & 43 & 56.8 & 9.2 & 9.3 & $0.57 \%$ \\
\hline 50 & 94 & 26.7 & 63.7 & 2 & 24 & 52 & 74 & 8.7 & 10.0 & $12.98 \%$ \\
\hline 43 & 88 & 9.7 & 3.6 & 2 & 24 & 44 & 40.21 & 10.3 & 10.1 & $2.46 \%$ \\
\hline 25 & 92 & 10.3 & 3.8 & 1 & 5 & 31 & 53.06 & 5.4 & 5.0 & $9.00 \%$ \\
\hline 30 & 82 & 4.7 & 8.8 & 0 & 48 & 61 & 84.4 & 5.4 & 6.5 & $16.54 \%$ \\
\hline
\end{tabular}

Tab.3 Random forest classification data

\begin{tabular}{|c|c|c|c|c|c|c|c|c|c|c|c|}
\hline Age(year) & $\begin{array}{l}\text { Course of } \\
\text { disease(month) }\end{array}$ & $\begin{array}{l}\text { Degree } \\
\text { of } \\
\text { goiter }\end{array}$ & $\mathrm{HR}$ & $\begin{array}{l}\text { Eye } \\
\text { sign }\end{array}$ & FT3(pmol/L) & FT4(pmol/L) & $\begin{array}{l}\text { 3-hour } \\
\text { iodine } \\
\text { uptake } \\
\text { rate }(\%)\end{array}$ & $\begin{array}{l}24- \\
\text { hour } \\
\text { iodine } \\
\text { uptake } \\
\text { rate(\%) }\end{array}$ & TW(g) & $\begin{array}{l}\text { Dose } \\
\triangle 10^{8} \mathrm{~Bq} \rrbracket\end{array}$ & Prognosis \\
\hline 49 & 3 & 3 & 92 & 0 & 10.19 & 2.69 & 38.11 & 53.63 & 46.74 & 8.78 & 1 \\
\hline 67 & 8 & 2 & 108 & 0 & 65.55 & 6.25 & 53.3 & 65.5 & 28.44 & 5.4 & 1 \\
\hline 28 & 6 & 3 & 88 & 0 & 12.90 & 1.53 & 73.9 & 39.5 & 74.65 & 19.6 & 0 \\
\hline 28 & 10 & 1 & 84 & 0 & 17.00 & 39.46 & 33.3 & 54.7 & 49.03 & 7.1 & 1 \\
\hline 27 & 5 & 3 & 100 & 1 & 6.76 & 1.48 & 90.23 & 86.34 & 60.38 & 5.4 & 0 \\
\hline 20 & 1 & 2 & 110 & 0 & 19.21 & 7.82 & 73.55 & 98.31 & 45.7 & 4.5 & 1 \\
\hline 41 & 8 & 1 & 92 & 0 & 9.83 & 4.06 & 31.87 & 56.36 & 23.59 & 4.6 & 1 \\
\hline 21 & 6 & 2 & 84 & 1 & 13.44 & 4.20 & 69.35 & 80.19 & 44.04 & 4.6 & 1 \\
\hline 55 & 14 & 3 & 100 & 0 & 20.00 & 7.77 & 81.22 & 74.2 & 158.67 & 11.2 & 0 \\
\hline 38 & 72 & 2 & 96 & 0 & 20.00 & 10.00 & 40.14 & 53.07 & 36.93 & 7.3 & 1 \\
\hline
\end{tabular}


Figures
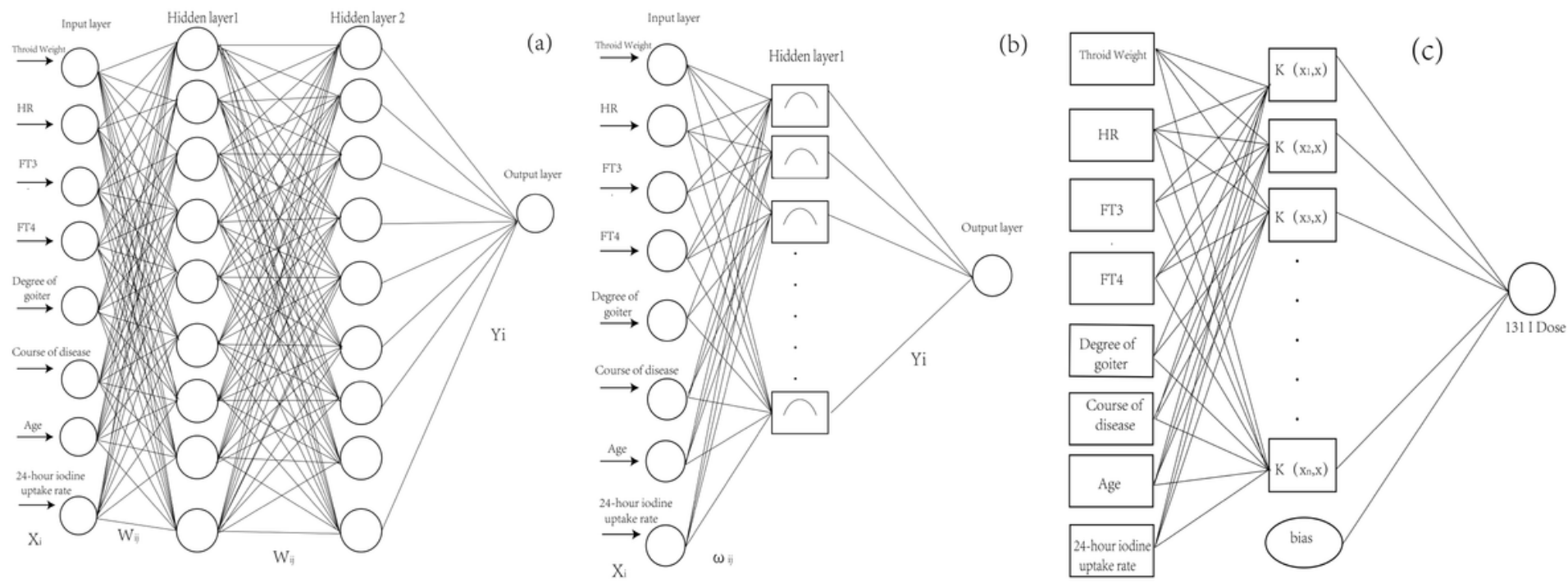

\section{Figure 1}

The topology of the neural network model with structure 8-9-9-1 used in this paper

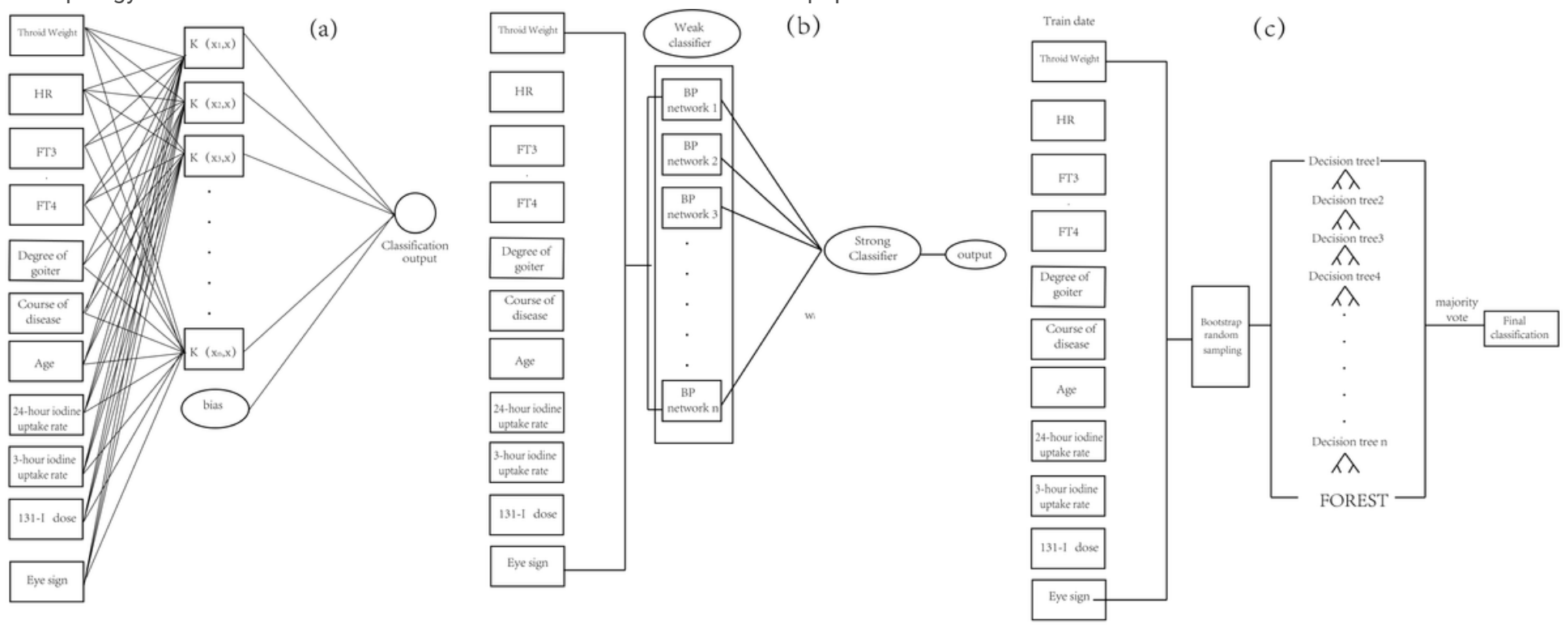

Figure 2

In this paper, "newrb" code in MATLAB is used to establish neural network, and "SPREAD" parameter set to 0.12. 


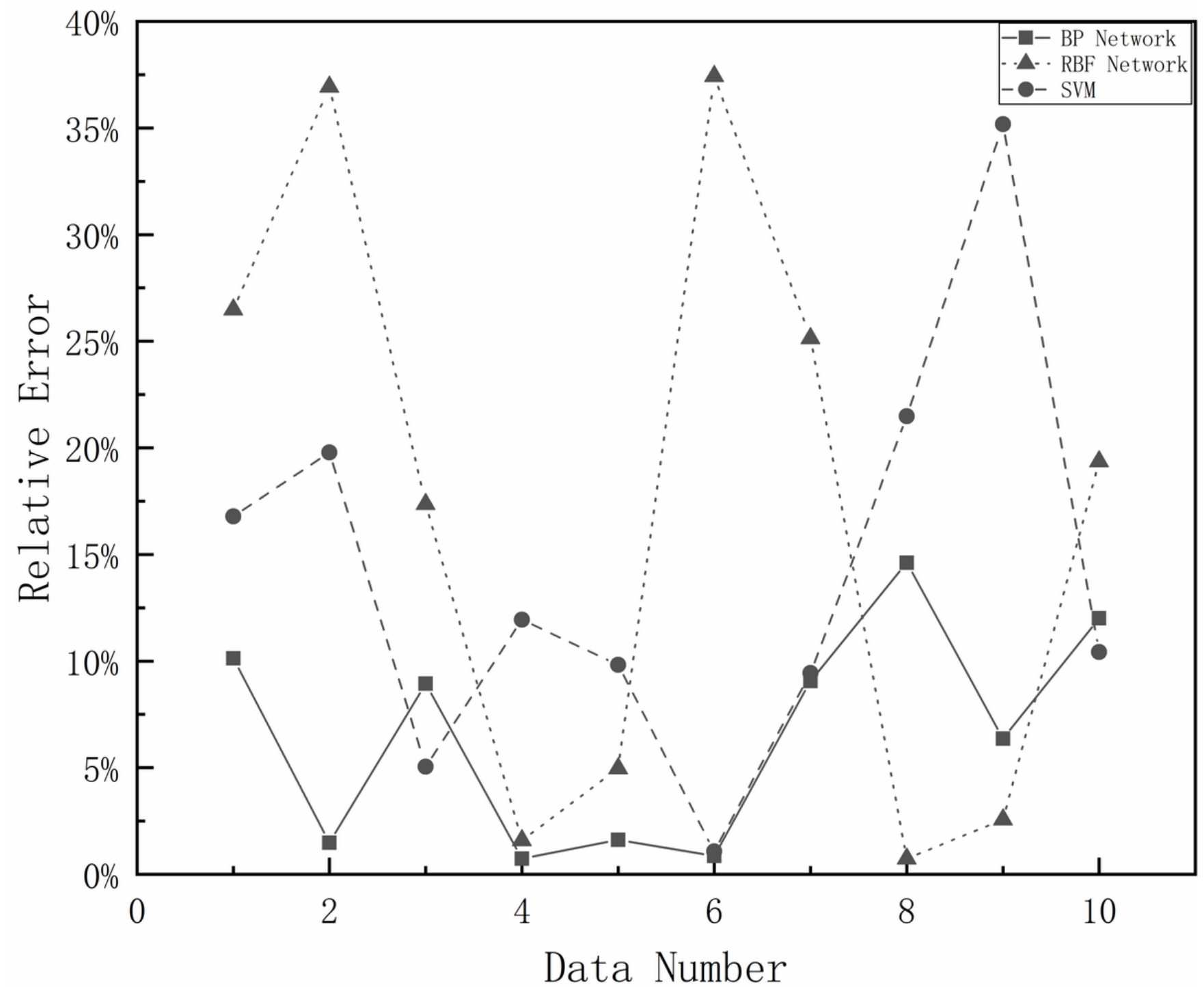

Figure 3

The predicted values of the three models are very close to the clinical data values, and the prediction ability is very good.

(A)

(B)
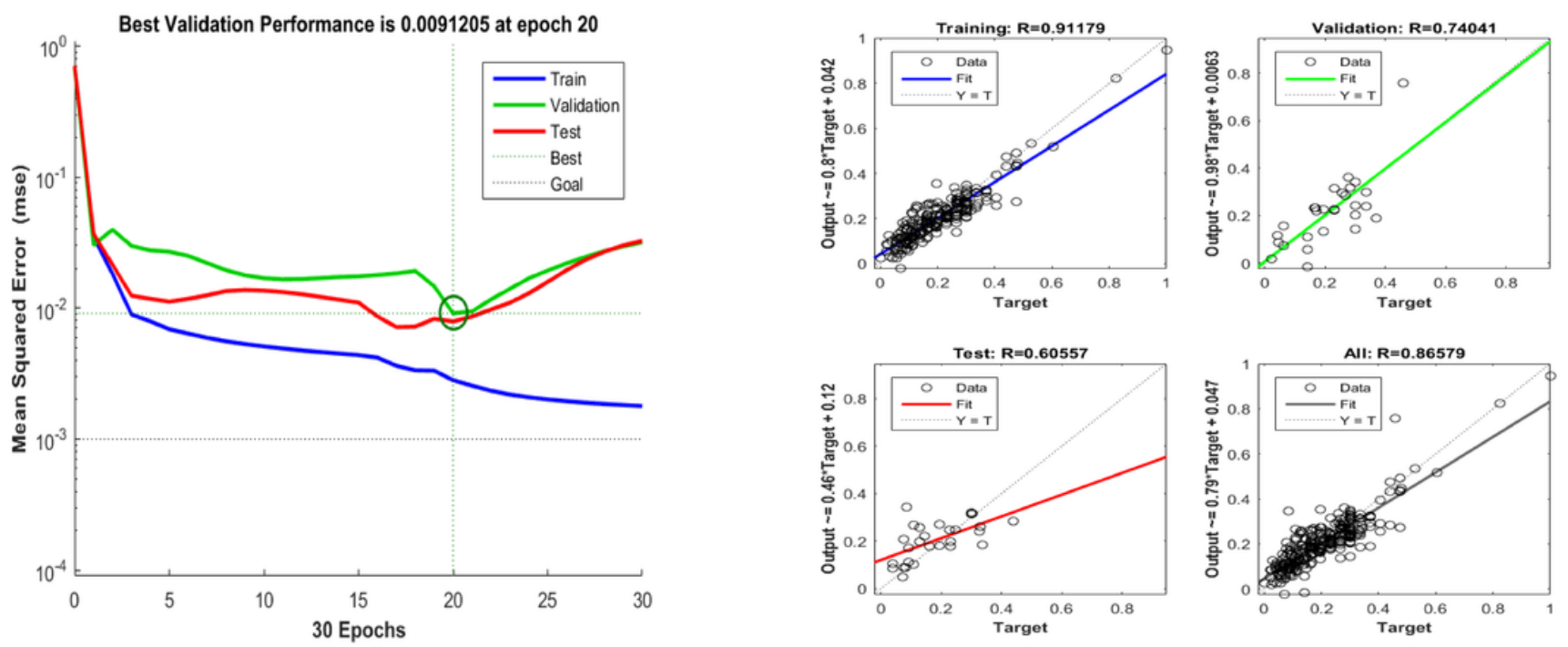
Figure 4

The overall training process of BP neural network is shown in Fig. 4. The data is divided into three parts by Matlab: "training", "validation" and "test". The "training" sample is used to train the network, "validation" sample is used to debug network parameters, and the "test" sample is used as evaluation to test the fitting effect. "Goal " is set by the "net trainparam Goal" parameter, which represents the expected minimum error". "Best" is the error accuracy actually obtained after training [24].

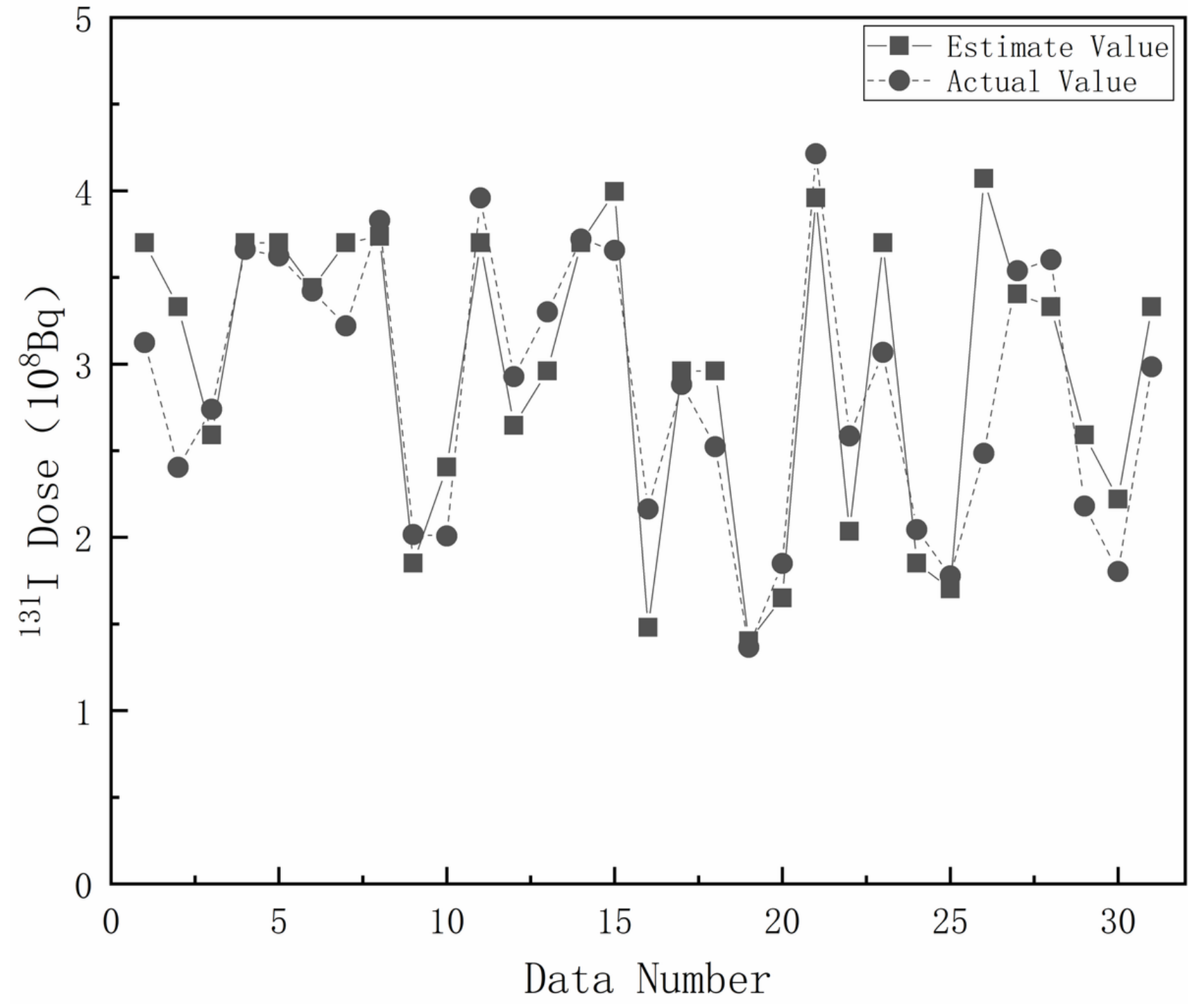

Figure 5

The comparison results between the output values and clinical data 


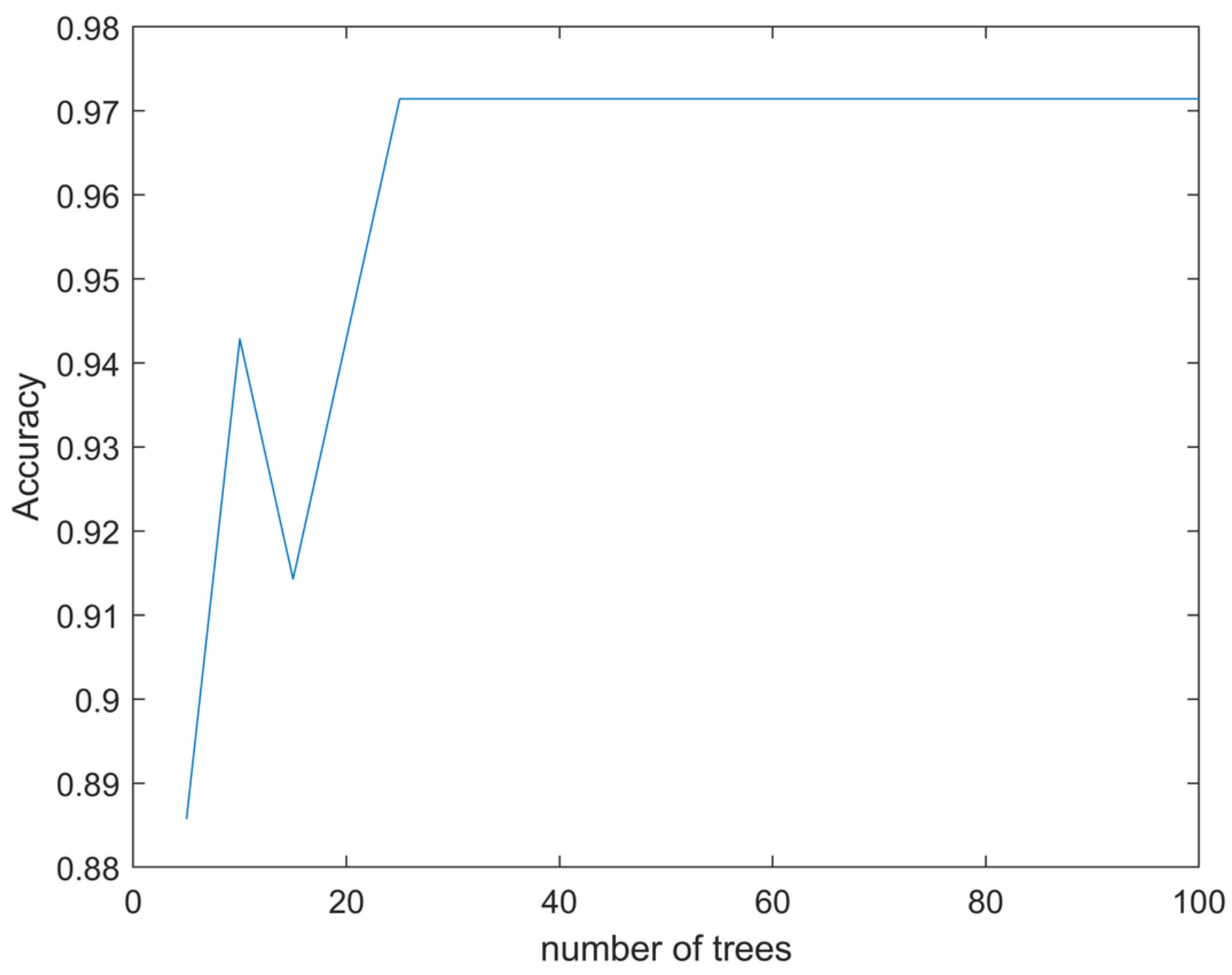

Figure 6

353 groups of all samples were divided into training set and test set according to the ratio of 9:1 for training. In order to obtain the optimal number of decision trees, this study compared the number of 5-100 decision trees. 